\title{
HIGH ENERGY OPERATION OF LEP2
}

\author{
S. Myers (for the LEP2 team), CERN, Geneva, Switzerland
}

\begin{abstract}
The LEP collider was operated during 1998 for the first time at a beam energy of $94.5 \mathrm{GeV}$. The 272 superconducting (sc) cavities were operated at an average gradient of more than $6 \mathrm{MV} / \mathrm{m}$. The high current in short bunches caused severe higher order mode heating of components. Nevertheless the machine proved very reliable with excellent performance. The maximum integrated luminosity over a 24 hour period exceeded $3.5 \mathrm{pb}^{-1}$ and the vertical beam-beam shift parameter reached more than .075 in three of the interaction regions with a record of .08 in the fourth. The present and future performance and limitations of the machine are reviewed as well as some of the crucial technical systems. During 1999 attempts will be made to increase the beam energy to around $100 \mathrm{GeV}$ with the help of an additional 16 sc cavities and by operating at gradients around $7 \mathrm{MV} / \mathrm{m}$.
\end{abstract}

\section{INTRODUCTION}

The CERN Large Electron Positron (LEP) collider is a $26.6 \mathrm{~km}$ circumference $\mathrm{e}^{+} \mathrm{e}^{-}$storage ring which has, until the end of 1995, operated with 4 to 12 bunches per beam in an energy range of 20 to $50 \mathrm{GeV}$ [1].

The first superconducting cavities for the LEP2 upgrade were installed in 1993. The complete installation of 256 Niobium film cavities and 16 sheet Niobium was completed early in 1998. By using up all remaining spare components and purchasing some missing parts a further 16 cavities have been produced and installed in the LEP tunnel in early 1999 [2],[3]. It is foreseen to operate LEP2 at the highest energies compatible with high integrated luminosity during 1999, and in 2000 to operate at the highest possible energy. Operation of LEP2 will be halted towards the end of 2000 to allow the preparation of the tunnel for the installation and operation of the LHC collider.

\section{HIGHLIGHTS OF 1998}

The integrated luminosity for 1998 (as compared with the four previous years) is shown in Fig 1. Clearly 1998 was highly successful, producing more than a factor of 2.5 higher than any previous year.

1998 was the first year which allowed operation of LEP2 for physics at high energies over a long uninterrupted period (around 120 days). The initial physics operation was performed with a new optics configuration with $102^{\circ} / 90^{\circ}$ phase advance per cell in the horizontal/vertical plane. This optics was designed to produce higher specific luminosity due to the inherently smaller horizontal emittance and a

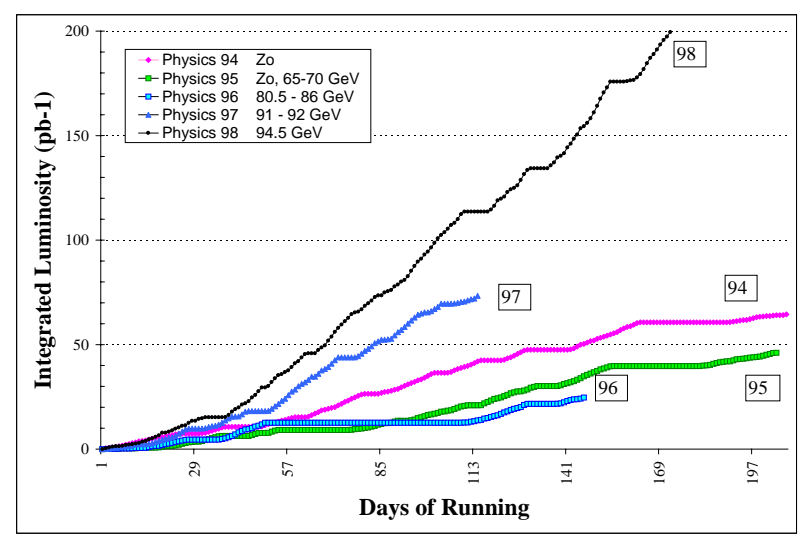

Figure 1: Integrated Luminosity during 1998

higher single bunch threshold for the transverse mode coupling instability due to the reduction in the effective transverse impedance produced by stronger focusing in the vertical plane. High tune optics also provide higher beam energy for the same RF voltage due to the reduction in the "overvoltage factor" brought about by the higher $\gamma_{t}$. In addition, at very high energies the natural emittance remains below the maximum value of $45 \mathrm{~nm}$ allowed in LEP for reasons of background and collimation. A detailed description of the advantages of this optics will be presented at this conference [4].

The main disadvantage with this optics is associated with the low energy running that is required for calibration of the detectors on the $Z^{0}$ peak. The inherent low emittance causes the beam beam limit to be reached at moderately low bunch intensities and therefore reduces the peak luminosity and lengthens the time needed to reach the required integrated luminosity of $2.5 \mathrm{pb}^{-1}$.

The strategy for defining the maximum energy of LEP is as follows. The all-out maximum voltage available is derived from the sum of

- the Niobium film sc cavities at their design value of $6 \mathrm{MV} / \mathrm{m}$ (256 in total in1998)

- the sheet Niobium cavities at 5MV/m (16 in total)

- and the room temperature copper cavities.

From this all-out maximum, the "operational" voltage is evaluated by subtracting $4 \%$ (for sick cavities) along with a further $160 \mathrm{MV}$, which is the voltage reduction which would result from the failure of 2 klystrons each of which feed 8 cavities (at $6 \mathrm{MV} / \mathrm{m}$ ). Knowing this operational voltage, the details of the optics, the value of the damping partition number, and assuming a quantum lifetime of 15 hours allows evaluation of the maximum beam energy. During 
physics, the cavities are operated at their all-out maximum (sometimes minus $4 \%$ ) thereby permitting the simultaneous failure of 2 klystrons without incurring total beam loss. The peak energy value was set to $94.5 \mathrm{GeV}$ for 1998 and was maintained at this value throughout the year. A single beam was accelerated to a maximum energy of $96.5 \mathrm{GeV}$ during a test run.

After initial conditioning and commissioning, the RF system behaved with remarkable reliability at (and sometimes above) its design gradient and with a total beam current of $6 \mathrm{~mA}$. The beam current was limited to this value by Higher Order Mode heating of the cavity control antennae (see later) and for normal operation the cavity conditions were optimized for this intensity value. Although the total current was limited, record peak luminosities of $1.0 \times 10^{32}$ $\mathrm{cm}^{-2} \mathrm{~s}^{-1}$ were reached due to the very small emittance ratio of $1 \%$ achieved (refer to Fig 3 ). The maximum integrated luminosity reached in a floating 24 hour period was $3.5 \mathrm{pb}^{-1}$ and $3.2 \mathrm{pb}^{-1}$ for a calendar day (see Fig 2).

On the beam dynamics front, the vertical beam-beam tune shift $\left(\xi_{y}\right)$, as measured from the average of the luminosities in the four detectors, reached values $\sim .075$ (see Fig 3) with a peak of $\sim .080$ in one of the collision points. Even at these elevated values there was little sign of saturation with $\xi_{y}$ increasing almost in direct proportion to the bunch current. Nevertheless such very high values of $\xi_{y}$ can only be achieved and maintained by very careful fine tuning of the lattice parameters (tunes, betatron coupling, global dispersion, local dispersion at the interaction points and the RF stations, etc.). This fine tuning is performed continuously during the physics data taking and successful manipulations are retained for future fills ("golden settings").

\section{PERFORMANCE LIMITATIONS IN 1998}

\subsection{Energy Calibration}

In LEP the accuracy of the measurement of the beam energy is of crucial importance for the precision of the physics. For LEP1 the beam energy is measured around the $\mathrm{Z}^{0}$ equivalent energy by transverse resonant depolarization [5]. For LEP2 it is likely that the polarization level at high energies will be too small to be measured. Hence the beam energy at $\mathrm{W} \pm$ must be estimated by extrapolation from beam energy measurement using resonant depolarization at lower energies [6]. The extrapolation is performed by the use of 16 NMR probes situated in a number of LEP dipoles, and the total bending field as measured by the flux loop. Clearly the accuracy of the extrapolation depends on the energy range over which the precise depolarisation measurements can be performed. In 1998, for the first time, successful calibrations were performed at four different energies in the range 41 to $61 \mathrm{GeV} /$ beam and in the same machine run. A second similar calibration was performed later in the year. Following this extension of the range, the re-

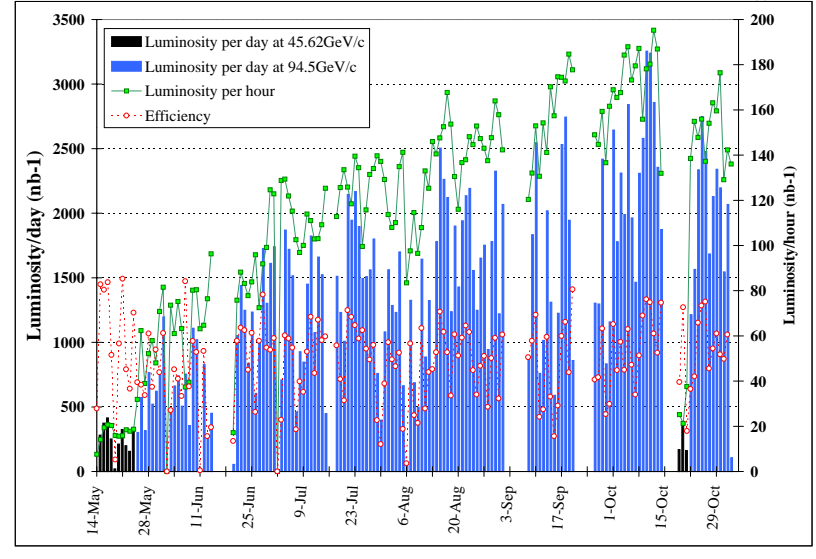

Figure 2: Daily and Hourly Luminosity during 1998

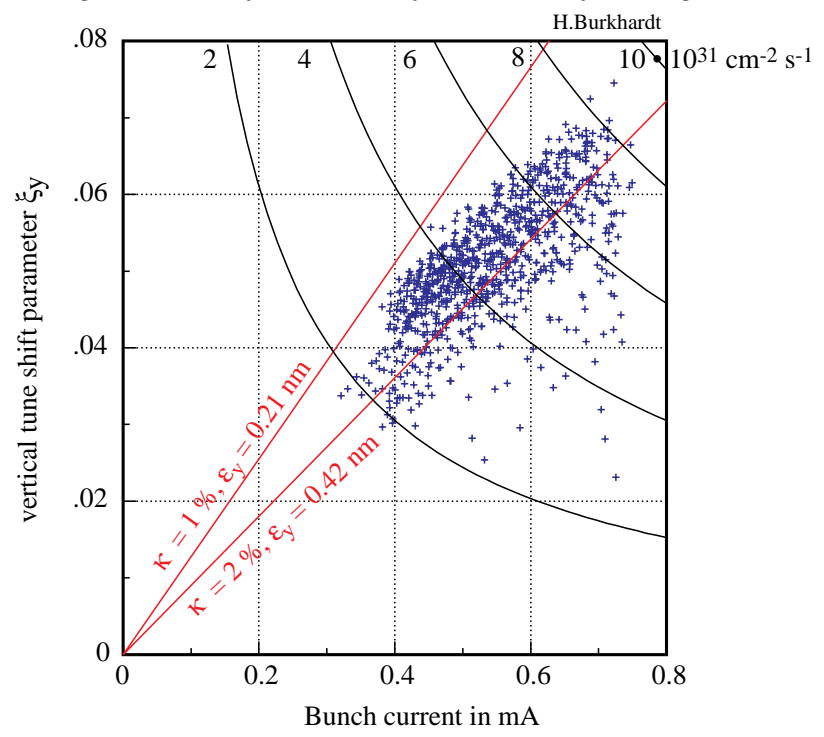

Figure 3: Vertical Beam-beam strength parameter $\left(\xi_{y}\right)$ as a function of bunch current.

maining systematic error of around $\pm 20 \mathrm{MeV}$ comes from discrepancies between the NMR and the flux loop. In order to reduce these errors to around $\pm 10 \mathrm{MeV}$ it is planned in 1999 to attempt resonant depolarisation at even higher energies. In addition a new spectrometer device [7] will be made available in 1999 as an additional cross-check.

Other techniques for energy calibration based on the energy loss per turn and the relationship between the synchrotron tune and the RF voltage were also tried [8] for the first time in 1998. These tests produced some promising results and will be pursued in 1999.

\subsection{Limitation due to cryogenic power}

In 1998, LEP2 was reliably operated with 4 cryogenic stations each having a maximum cooling capacity of around $12 \mathrm{~kW}$. After subtracting the static losses associated with each of these stations the remaining "dynamic" power for cooling the cavities and any beam losses is $\sim 6.2 \mathrm{~kW}$. The required power for cooling of the cavities is 


$$
P_{\text {cav }} \propto \frac{\text { Gradient }^{2}}{Q_{\text {cav }}}
$$

where $Q_{\text {cav }}$ is the cavity quality factor which decreases with increasing gradient.

The second contribution to the cryogenic power is due to higher order mode losses and can be characterised by

$$
P_{c m}=Z I_{t o t}^{2}=\frac{R_{m}\left(\sigma_{s}\right)}{n_{b} k_{b}} I_{t o t}^{2}
$$

where $n_{b} k_{b}$ is the total number of bunches in both beams and $R_{m}\left(\sigma_{s}\right)$ is the bunch length dependent impedance related to the HOM loss factor.

Measurements of the increase in the cryogenic power as a function of beam current allowed (in 1997) $R_{m}$ to be evaluated as $16 \mathrm{M} \Omega[9]$.

Use of equations (1) and (2) with the known maximum dynamic power and the measured value for $R_{m}$ allows evaluation of the maximum permissible current as a function of the cavity gradient (beam energy). This procedure indicated a total current of around $6.7 \mathrm{~mA}$ at an average gradient of $6 \mathrm{MV} / \mathrm{m}$ which corresponds to a beam energy of $94.5 \mathrm{GeV}$.

\subsection{Heating and Damage to Cavity Antennae Cables}

During the winter shutdown 1997/1998 it was discovered that many of the cables attached to the cavity electromagnetic field probes were electrically damaged and some were severely burnt and open circuited. These cables transmit the signals used to control the field and phase of the cavities. The damage always occurred at a location where the cables had been routed through the cavity superinsulation. Subsequent studies showed that the spurious cavity (produced by the housing inside which the antennae were lodged) was responsible for significantly enhancing the coupling of the high frequency beam power to the antennae.

Later investigation also showed that the heating of the cables explained the beam-related cryogenic losses as measured via equation (2).

In order to reduce the risk of overheating a large number of cables during the 1998 run it was decided that the cable temperature should be limited. It was however impossible to obtain an accurate measurement of the temperature in situ and in the presence of beam. For this reason a power/temperature calibration test was done in the laboratory [10] in order to evaluate the power at which the cable temperature reached the upper limit of its specifications. Following this calibration the power induced in the cables as measured in the tunnel was limited to $8 \mathrm{~W}$ which corresponded to the maximum temperature permissible. Throughout the 1998 run every effort was made to increase the beam intensity without exceeding the $8 \mathrm{~W}$ limit. This involved the maximization of the bunch length throughout the ramp by the use of wiggler magnets and $\mathrm{Q}_{S}$ variations. The maximum beam intensity was ultimately limited to around $6 \mathrm{~mA}$.

Towards the end of the 1998 run, the limit was increased to $8.5 \mathrm{~W}$ and the rate of loss of cables increased dramatically. Hence for the last few weeks the intensity was more severely constrained in order to survive with a full complement of cavities to the scheduled end of the run.

\section{FUTURE PERFORMANCE AND LIMITATIONS}

\subsection{Higher Order Mode Heating}

Replacement of RF Antennae Cables By the end of the 1998 run, even with the total beam current limited to $6 \mathrm{~mA}$, more than 30 cavity antennae cables had been burned. In September 1998 an in situ test was scheduled to replace the existing antennae cables on a single RF module (4 cavities) by thicker ones which were not routed through the superinsulation. Following the success of this test a crash programme was launched to replace every single antennae cable in LEP during the winter shutdown. This programme was successfully completed by mid February 1999. Consequently the level of the intensity limitation due to antennae cable heating will in the future be significantly higher than other intensity limitations.

A comparison of the power losses in the module equipped with the new cables showed that the beam related heat losses were reduced by nearly a factor of two (in equation (2), $R_{m}$ was reduced to around $8 \mathrm{M} \Omega$ ). This results from the better heat conducting properties of the newly installed cables.

\subsection{Cryogenics Upgrade}

It has been shown in section 3 that, with the existing $12 \mathrm{~kW}$ LEP2 cryogenic installation, the beam energy in LEP cannot be significantly increased beyond the 1998 values. In order to allow an increase in the operational energies it was decided in 1997 to upgrade the four cryogenic installations. The most economic way to perform these upgrades was to plan an early installation of part of the cryogenics system needed for the LHC magnets. In this way each of the four cryo plants could be upgraded to around $18 \mathrm{~kW}$ thereby leaving around $12 \mathrm{~kW}$ "dynamic" power: giving almost a factor of two increase.

\subsection{RF Gradients}

The over-riding limitation to the beam energy comes from the available total RF voltage which is simply the product of the active length of the cavities and the average gradient. During the 1998/1999 shutdown an additional 16 Niobium film sc cavities were installed in the LEP tunnel bringing the total to 272 with an additional $16 \mathrm{sc}$ cavities made from Niobium sheet.

The required average gradient of the installed sc cavities is plotted as a function of the beam energy in Fig 4. It is 


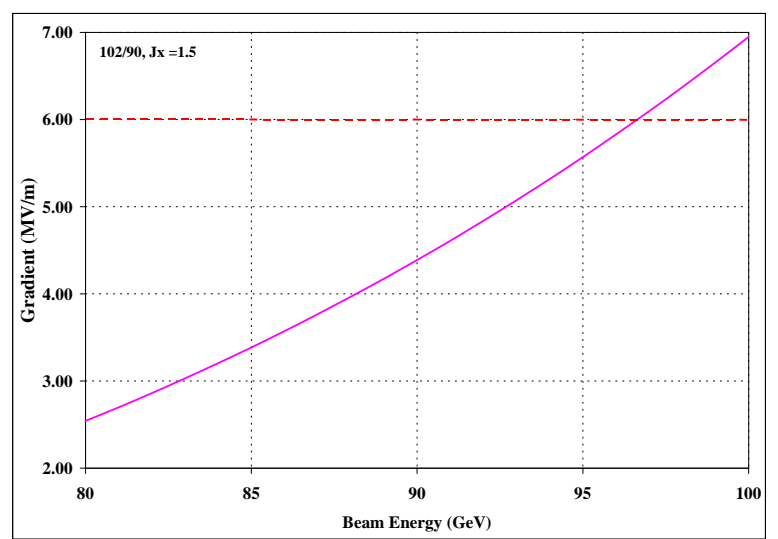

Figure 4: Required gradient in the $272 \mathrm{NbCu}$ cavities as a function of the beam energy $\left(102^{0} / 90^{\circ}\right.$ optics and $\left.J_{x}=1.5\right)$

clear that the design gradients of $6 \mathrm{MV} / \mathrm{m}$ must be increased to $7 \mathrm{MV} / \mathrm{m}$ in order to reach a beam energy of $100 \mathrm{GeV}$. The three main difficulties with increasing the cavity gradients are:

1. electron emission,

2. dispersion in the gradients about the average value,

3. mechanical oscillations driven by the electromagnetic pressure (ponderomotive oscillations).

Electron emission in the cavities. Early in 1997 after conditioning and processing the sc cavities up to their design value of $6 \mathrm{MV} / \mathrm{m}$, it was found that the radiation levels in some cavities remained high $(\leq 20 \mathrm{krads} / \mathrm{hour})$ when operating at $6 \mathrm{MV} / \mathrm{m}$. Early in 1998 , in order to reduce the radiation levels (at the nearby vacuum valves) to below around 10krads/hour, the cavities were conditioned by a mixture of pulsed and continuous processing at gradients between 6.5 and $7 \mathrm{MV} / \mathrm{m}$. Fig 5 shows that this operation was successful in that at average gradients of $6 \mathrm{MV} / \mathrm{m}$ the levels are all below 10krads/hour. However further augmentation of the gradients produced a large increase in the radiation (refer to to points at or above $7 \mathrm{MV} / \mathrm{m}$ in Fig 5 . Consequently, in order to operate around $7 \mathrm{MV} / \mathrm{m}$ with reduced radiation, it will be necessary to condition the cavities with a mixture of pulsed and continuous processing at fields between 7.5 and $8 \mathrm{MV} / \mathrm{m}$. Such high fields will be risky for the whole RF system, particularly the couplers and the cavities themselves.

Helium processing is used less frequently because of the time constraint and the inherent danger for the main couplers. Nevertheless Helium processing has and will continue to be used to recuperate "sick cavities".

Dispersion in cavity gradients. When an RF unit (8 cavities fed by one klystron) is operating at an average gradient per cavity of $6 \mathrm{MV} / \mathrm{m}$, the spread in the gradients is typically more than $\pm 1 \mathrm{MV} / \mathrm{m}$. Consequently some of the cavities in a unit are already being operated at $7 \mathrm{MV} / \mathrm{m}$. It

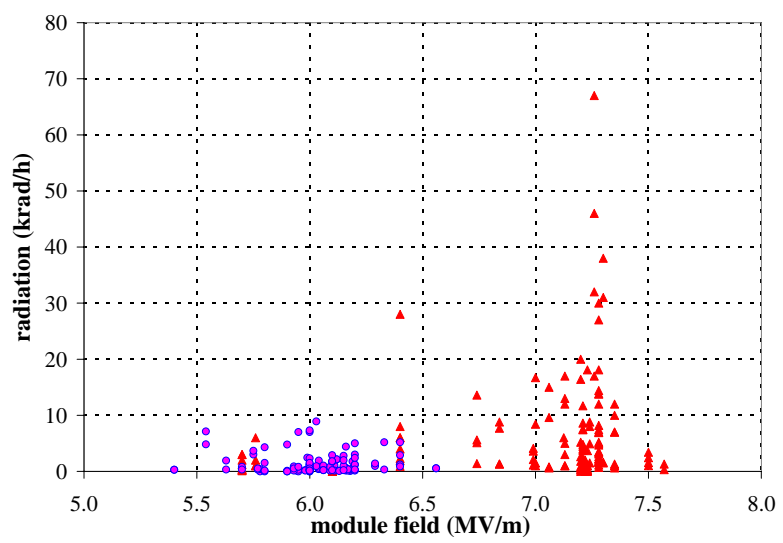

Figure 5: Measured radiation as a function of the gradient.

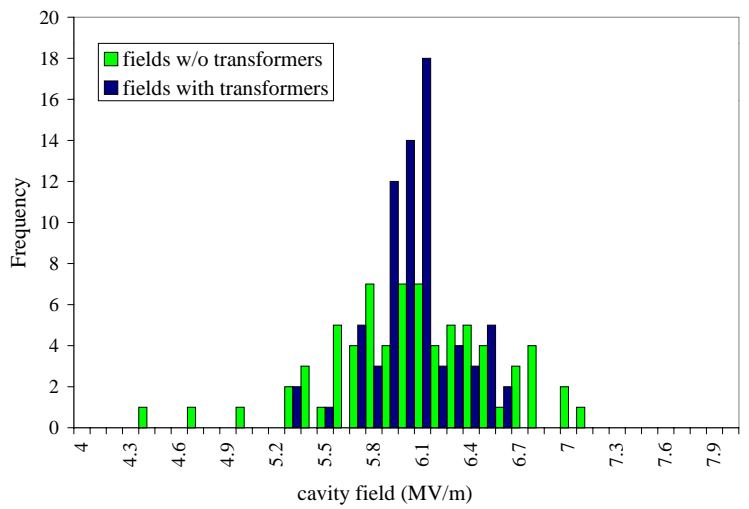

Figure 6: Dispersion in cavity gradients.

is clear that an average of $7 \mathrm{MV} / \mathrm{m}$ will be more easily obtained if the spread of gradients in the modules is significantly reduced.

The spread in the gradients results from waveguide differences, asymmetries in the power splitting and most importantly differences in the external $Q$ values. The latter can be improved by installing transformers in the waveguides. Fig 6 shows (for measurements made in a test sector) the spread in gradients for modules before and after being equipped with such transformers. Following these results an aggressive programme is on-going to equip the maximum number of cavities with such transformers.

Ponderomotive Oscillations Early operation of the LEP2 cavities showed that, at high beam currents and high gradients, the cavities oscillated mechanically at around $100 \mathrm{~Hz}$ which resulted in large gradient oscillations at the same frequency. The cause of these oscillations was later identified [11] as an instability driven by the combination of volume changes in the cavities, caused by the electromagnetic forces and the fact that the cavities are driven off-tune. The growth rate of the instability was shown to be proportional to the product of the gradient squared and the beam current. These oscillations have, until the end of 1998 been controlled by variation of the tuning of the cav- 


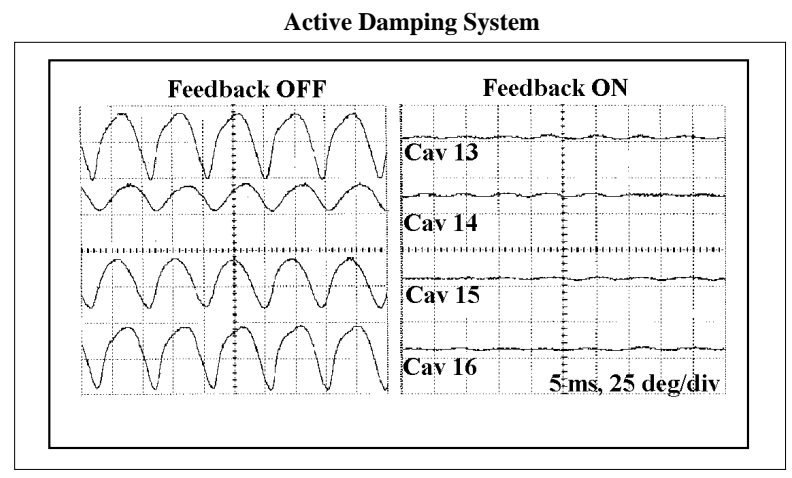

Unit 873_2, Vc=10.2 MV, Ib=5.2 $\mathrm{mA}$

Figure 7: Influence of the system for damping ponderomotive oscillations

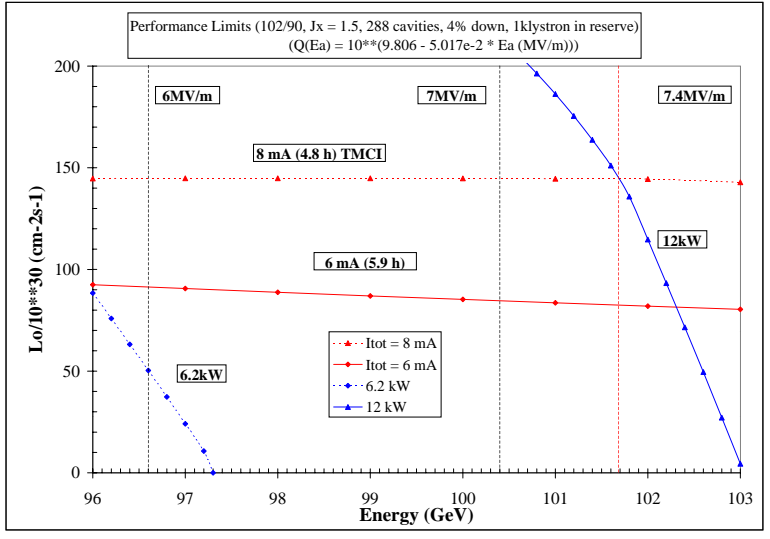

Figure 8: Luminosity and Energy Limitations

ities. During 1998 an automatic programme was used to find the optimum setting for each individual cavity. Unfortunately these settings are energy (gradient) and beam current dependent. During 1998 a new tuning/damping system was developed which feeds back on the cavity tuners from signals derived from the phase and amplitude of the cavity field. Fig 7 shows the damping of oscillations with this new system. A progressive installation of the new damping system will be carried out during 1999.

\section{PERFORMANCE IN 1999 AND 2000}

The addition of $16 \mathrm{sc}$ cavities, the replacement of the antennae cables and the upgrade of the cryogenics plants significantly increases the potential for LEP2. Fig 8 shows the performance limits in the luminosity against energy plane [12] [13]. The conditions used for this plot are those pertinent for 1999 and 2000, consequently they are not directly comparable with the previously stated limits for 1998.

Examination of this plot indicates that the most crucial limit to performance is given by the RF gradients. It is evident that, provided the gradients can be raised to values of $7 \mathrm{MV} / \mathrm{m}$ in a technically reliable way, the high luminosity physics will be possible at energies in the $100 \mathrm{GeV} /$ beam range.

\section{CONCLUSIONS}

The performance of the LEP collider was significantly improved for the 1998 run with higher energies and a very high integrated luminosity. The recent various technical upgrades combined with the detailed work carried out to allow higher RF gradients may allow operation at energies in the $100 \mathrm{GeV}$ range with high luminosities.

\section{ACKNOWLEDGEMENTS}

This paper reports the work done by a very large number of scientists and technicians who have dedicated a large fraction of their professional life to the successful design, construction, and operation of the LEP collider.

\section{REFERENCES}

[1] S. Myers; "LEP Status and Plans", Proc. of the US Particle Accelerator Conference and the 16th Int. Conf. on High Energy Accelerators, Dallas USA. (1995).

[2] K. Hübner; "LEP Present and Future Performance and Limitations", Proc. of the Sixth European Particle Conference (EPAC98), held in Stockholm, Sweden from 22 June until 26 June 1998, also CERN-SL-98-043-DI.

[3] S. Myers; "LEP2; Present and Future Performance and Limitations", Proc. of the 1997 US Particle Accelerator Conference (PAC'97), held in Vancouver, BC, Canada, from 12 May until 16 May 1997 . Publ. in Proceedings also CERNSL-97-022-DI.

[4] M. Meddahi et al. "Experience with a low emittance optics in LEP", paper presented to this conference.

[5] R. Assmann et al., "Calibration of centre-of-mass energies at LEP1 for precise measurements of Z properties", Eur. Phys. J. C6, 187-223 (1999) also CERN-SL/98-012.

[6] A. Blondel et al., "Evaluation of the LEP centre-of-mass energy above the W-pair production threshold", CERN-EP198-191 also CERN-SL/98-073, December 1998. Submitted for publication in Eur. Phys. J. C.

[7] B. Dehning, "The LEP Spectrometer", Proc. of the 1999 Workshop on LEP-SPS (Chamonix IX) held in Chamonix, France in January 1999.

[8] A-S. Müller, "Energy Calibration for the price of a Fig", Proc. of the 1999 Workshop on LEP-SPS (Chamonix IX) held in Chamonix, France in January 1999.

[9] Lep2000 Working Group, "LEP 2000 Status Report", CERN SL-98-011 DI, March 1998.

[10] G. Cavallari, private communication May 1998.

[11] D.Boussard, P. Brown, and J. Tückmantel, "Electroacoustic oscillations in the LEP SC. cavities" Proc. 5th European Particle Accelerator Conference, EPAC '96 held in Sitges, Barcelona, Spain on 10 to 14 Jun 1996, also CERN-SL-96017-RF.

[12] D. Brandt and S. Myers; "LEP Performance Limits revisited", CERN-SL-98-074-DI.

[13] D. Brandt, K. Hübner, and S. Myers; "On LEP Performance Limits in 1999 and 2000”, (CERN-SL-98-005-DI). 